\title{
Prevalence of Enteric Protozoan Oocysts with Special Reference to Sarcocystis cruzi among Fecal Samples of Diarrheic Immunodeficient Patients in Iran
}

\author{
Mahmoud Agholi, ${ }^{1,2}$, Shahrbanou Naderi Shahabadi ${ }^{3}$, Mohammad Hossein Motazedian4, Gholam Reza Hatam ${ }^{4, *}$ \\ ${ }^{1}$ HIV/AIDS Research Center, Fasa University of Medical Sciences, Fasa, Iran; ${ }^{2}$ Department of Microbiology, Fasa University of Medical Sciences, \\ Fasa, Iran; ${ }^{3}$ Department of Parasitology and Mycology, School of Medicine, Shiraz University of Medical Sciences, Shiraz, Iran; ${ }^{4 B a s i c}$ Sciences in \\ Infectious Diseases Research Center, Shiraz University of Medical Sciences, Shiraz, Iran
}

\begin{abstract}
The genus Sarcocystis is not usually considered as an important enteric pathogen in immune compromised patients. It might be expected that species for which humans are the final host (Sarcocystis hominis and Sarcocystis suihominis as well as possibly others) would be encountered increasingly often in immunodeficient persons. This study aimed to address how to detect and differentiate Sarcocystis oocysts and/or sporocysts from enteric protozoans in the diarrheal samples of immunodeficient patients in Shiraz, Iran. Diarrheal samples of 741 immunodeficient patients with recurrent persistent or chronic diarrhea were examined by microscopy and molecular biological analysis. Oocysts-positive samples were 68 Cryptosporidium spp., 9 Cystoisospora belli (syn. Isospora belli), 2 Cyclospora cayetanensis, and 15 microsporidia (Enterocytozoon bieneusi). Sarcocystis-like sporocysts found from a woman were identified as Sarcocystis cruzi through $18 \mathrm{~S}$ rDNA amplification and phylogenetic analysis. To the best of our knowledge, this is the first report of $S$. cruzi from a human.
\end{abstract}

Key words: Sarcocystis cruzi, PCR, sporocyst, diarrhea, human, AIDS

Intestinal parasites, both protozoa and helminths, are 2 main enteric pathogens in developing countries, and some of these are responsible for emerging and reemerging infections in immunodeficient patients [1-5]. Sarcocystosis is an intracellular protozoan parasitic disease caused by coccidia of the genus Sarcocystis [6]. Since the first report of Sarcocystis infection, more than 120 species have been identified in this genus [6].

Depending on the species of Sarcocystis, these parasites are causative agents of intestinal or muscular sarcocystosis in human beings [6]. Sarcocystis protozoa of the intestinal tract in humans as the final host (excretion of sporocysts with feces) have a similar life cycle. In humans, infection occurs from eating uncooked or poorly cooked meat of some livestock with infective stages (sarcocysts) and the sexual phase (gametogony) occurs in the intestinal mucosa [6,7]. In some countries, infection has potential public health importance, because humans may acquire infection by consumption of raw or under-

\footnotetext{
- Received 23 October 2015, revised 18 April 2016, accepted 25 April 2016.

*Corresponding author (Hatam908@yahoo.com; hatamghr@sums.ac.ir)

(c) 2016, Korean Society for Parasitology and Tropical Medicine

This is an Open Access article distributed under the terms of the Creative Commons Attribution Non-Commercial License (http://creativecommons.org/licenses/by-nc/4.0) which permits unrestricted non-commercial use, distribution, and reproduction in any medium, provided the original work is properly cited.
}

cooked beef or pork causing intestinal sarcocystosis [6]. For example, Sarcocystis-associated acute enteritis was reported in 6 cases in Thailand. Sarcocystis is not usually considered as an important enteric pathogen in immunocompromised patients but a report has been published on intestinal sarcocystosis-associated cholecystitis in Iran [8].

Although there are several reports about the prevalence of intestinal sarcocystosis in humans from different regions of the world, there is no published data about identified species causing intestinal infections using molecular methods in Iran. The primary aim of the present study was detection and differentiation of Sarcocystis oocysts and/or sporocysts from enteric protozoans in diarrheal samples of immunodeficient patients in Shiraz, Iran.

In this study, fecal samples were collected from $741 \mathrm{immu}-$ nodeficient individuals (493 males and 248 females), including 387 patients with HIV/AIDS; 187 patients with malignancy, especially while on chemotherapy; 124 of those who had received either a solid organ or a bone marrow transplant; 43 individuals with prolonged corticosteroid therapy or hypogammaglobulinemia with recurrent persistent or chronic diarrhea from October 2009 to October 2014. The stool samples collected in clean plastic containers were transferred to the 
Laboratory of Intestinal Protozoology at Shiraz University of Medical Sciences.

Whenever possible, more than 1 fecal sample was collected from each patient. After recording the consistency of feces (watery or loose), color, gender, and age (aged 3-94) as well as underlying diseases, each sample was examined by direct wet mount, formalin-ethyl acetate concentration, and modified acid-fast or acid-fast trichrome stains. In the fecal samples containing Sarcocystis, sporocysts were floated using Sheather's sugar flotation procedure to harvest sporocysts from stool debris. To remove the sucrose solution and to prepare the sporocysts for lysis, the harvested sporocysts were diluted in $1 \mathrm{ml}$ sterile tap water, centrifuged and washed in saline solution 2 times repeatedly. The washed sporocysts were stored at $4^{\circ} \mathrm{C}$ until use for molecular studies.

For molecular identification, DNA was extracted from harvested sporocysts using proteinase $\mathrm{K}$ digestion, and phenolchloroform purification followed by ethanol precipitation method as previously described. DNA extracted was then frozen at $-20^{\circ} \mathrm{C}$ until use for molecular studies. A fragment of the $18 \mathrm{~S}$ rDNA gene was amplified using a semi-nested PCR procedure, as previously described [9] and reported in previous in- vestigations in the laboratory [8]. The outer primers were $2 L$ (GGATAAACCGTGGTAATTCTATG) and 2H (ACCTGTTATTGCCTCAAACTTC) and the inner primers were $2 L$ and $3 H$ (GGCAAATGCTTTCGCAGTAG) $[8,9]$. The templates were subjected to 30 amplification cycles $\left(94^{\circ} \mathrm{C}\right.$ for $40 \mathrm{sec}, 53^{\circ} \mathrm{C}$ at primary PCR and $56^{\circ} \mathrm{C}$ at secondary PCR for $60 \mathrm{sec}, 72^{\circ} \mathrm{C}$ for $100 \mathrm{sec}$ at primary PCR and $80 \mathrm{sec}$ at secondary PCR) followed by 1 cycle $7 \mathrm{~min}$ at $72^{\circ} \mathrm{C}$ and held at $4^{\circ} \mathrm{C}[8,9]$. Amplification product was analyzed by electrophoresis through a $2 \%$ agarose gel for the Sarcocystis-specific PCR. PCR product was directly sequenced with the set of primers $2 \mathrm{~L}$ and $3 \mathrm{H}$ used for the sec-

Table 1. Prevalence of enteric protozoan oocysts and microsporidia in diarrheic immunodeficient patients $(n=741)$

\begin{tabular}{lrl}
\hline Protozoa & No. of positive samples (\%) \\
\hline Cryptosporidium spp. & $68(9.2)$ \\
Cystoisospora belli & $9(1.2)$ \\
Cyclospora cayetanensis $^{*}$ & $2(0.3)$ \\
Sarcocystis $^{*}$ & 1 & $(0.1)$ \\
Microsporidia & $15(2.0)$ \\
Negative & $646(87.2)$ \\
\hline
\end{tabular}

All samples were double examined by microscopy and semi-nested PCR $[2,3]$.
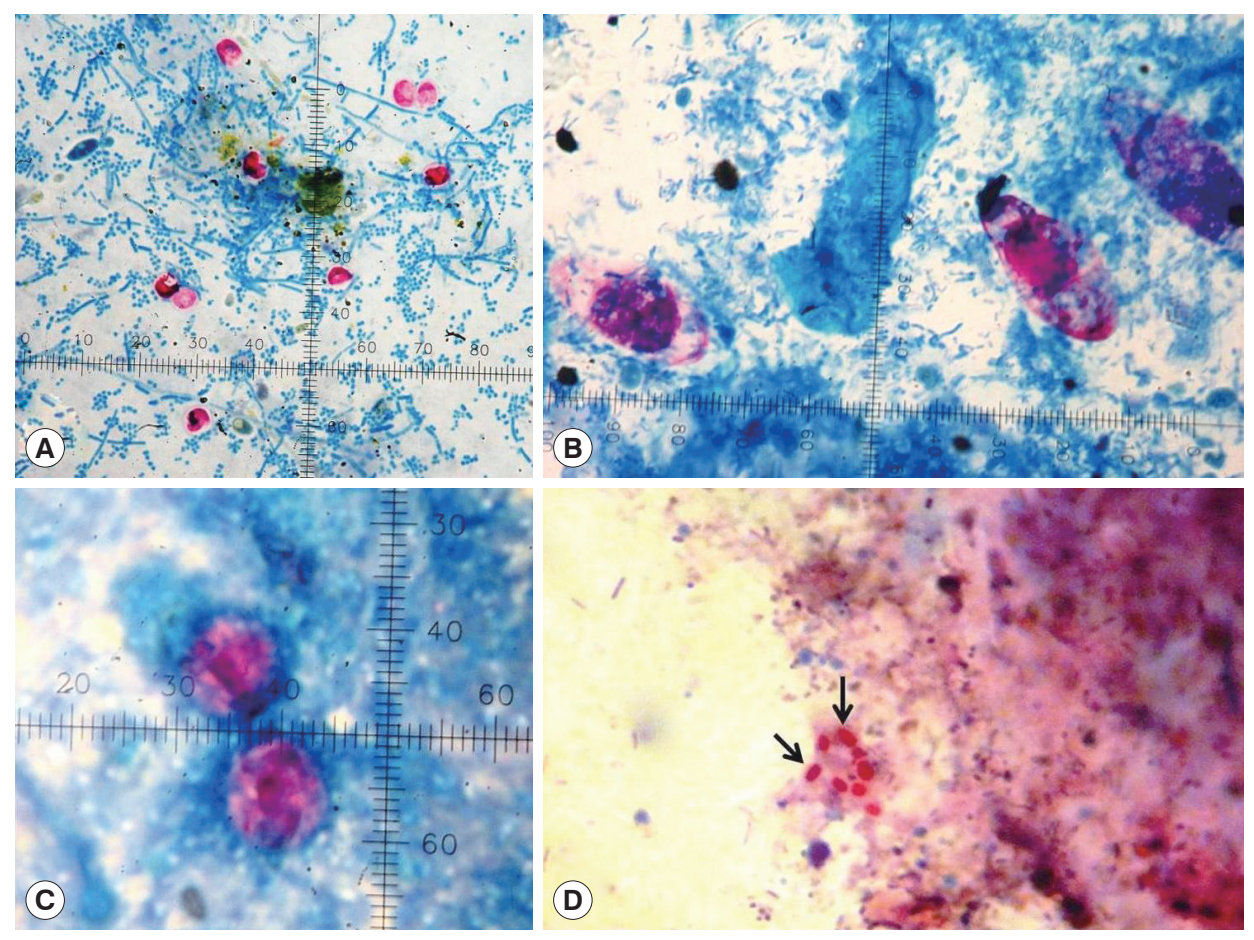

Fig. 1. Modified Kinyoun's acid fast staining showing enteric protozoan oocysts. (A) Cryptosporidium species (4-6 4 m). (B) Cystoisospo-

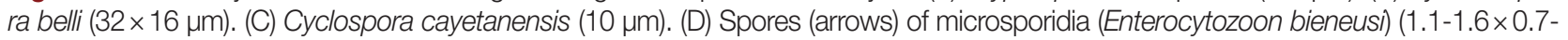
$1.0 \mu \mathrm{m})$. 
ondary step. The sequence was compared with sequences in the GenBank database by BLAST analysis. For better understanding of relationship among $S$. cruzi and other species in the genus Sarcocystis, a phylogenetic analysis of $18 \mathrm{~S} \mathrm{rDNA}$ was performed using CLC Sequence Viewer 6 software (http:// www.clcbio.com). Neospora caninum was used as the outgroup. All the microscopic-negative fecal samples were also ruled out using DNA extraction and the same primers [8,9]. In addition to microscopic examinations, semi-nested PCR analyses were performed to confirm whether isolated oocysts were of other protozoans (e.g., Cryptosporidium, Cystoisospora belli, and $\mathrm{Cy}$ clospora cayetanensis), as previously reported [2].

Microscopic and PCR examinations on the patients' samples revealed oocysts of Cryptosporidium spp., C. belli (syn. Isospora belli), C. cayetanensis, and microsporidia (Enterocytozoon bieneusi) in 68, 9, 2, and 15 cases (Fig. 1), respectively (Table 1). In addition, scanty sporulated sporocysts of Sarcocystis sp., each containing 4 sausage-shaped sporozoites and a mass of refractile granules called residual body were observed in the stool specimen collected from a patient (Fig. 2A). Each sporocyst was ovoid in shape and measured 14.5-17.0 $\times 9-11 \mu \mathrm{m}$ in size
(Fig. 2A).

The semi-nested PCR produced a 850 bp amplicon (Fig. 2B). Sequence of the PCR product was close to $S$. cruzi retrieved from GenBank. With these 2 findings, the sporocysts found from a patient were identified as those of Sarcocystis cru$z i$. Nucleotide sequence of this parasite was deposited in the GenBank database under accession no. KR136315. Sarcocystis DNA was not detected from any of microscopically negative samples.

Sarcocystis-positive diarrheal sample was from a woman with AIDS and a CD4 $4^{+}$count of less than 100 cells/ $/ \mu l$. Prior to fecal microscopic examination in author's laboratory, she had a history of intermittent diarrhea that was variably associated with nausea, vomiting, loss of appetite, and weight loss for 6 months. As the patient had intermittently experienced this diarrhea, she had been previously undergone cholecystectomy, and repeated histopathological investigation of the gallbladder biopsy had demonstrated acalculous cholecystitis with nonspecific inflammations. An ultrasound scan of the right upper quadrant had revealed thickening of the anterior portion of the gallbladder wall with no stones, pericholecystic fluid, or
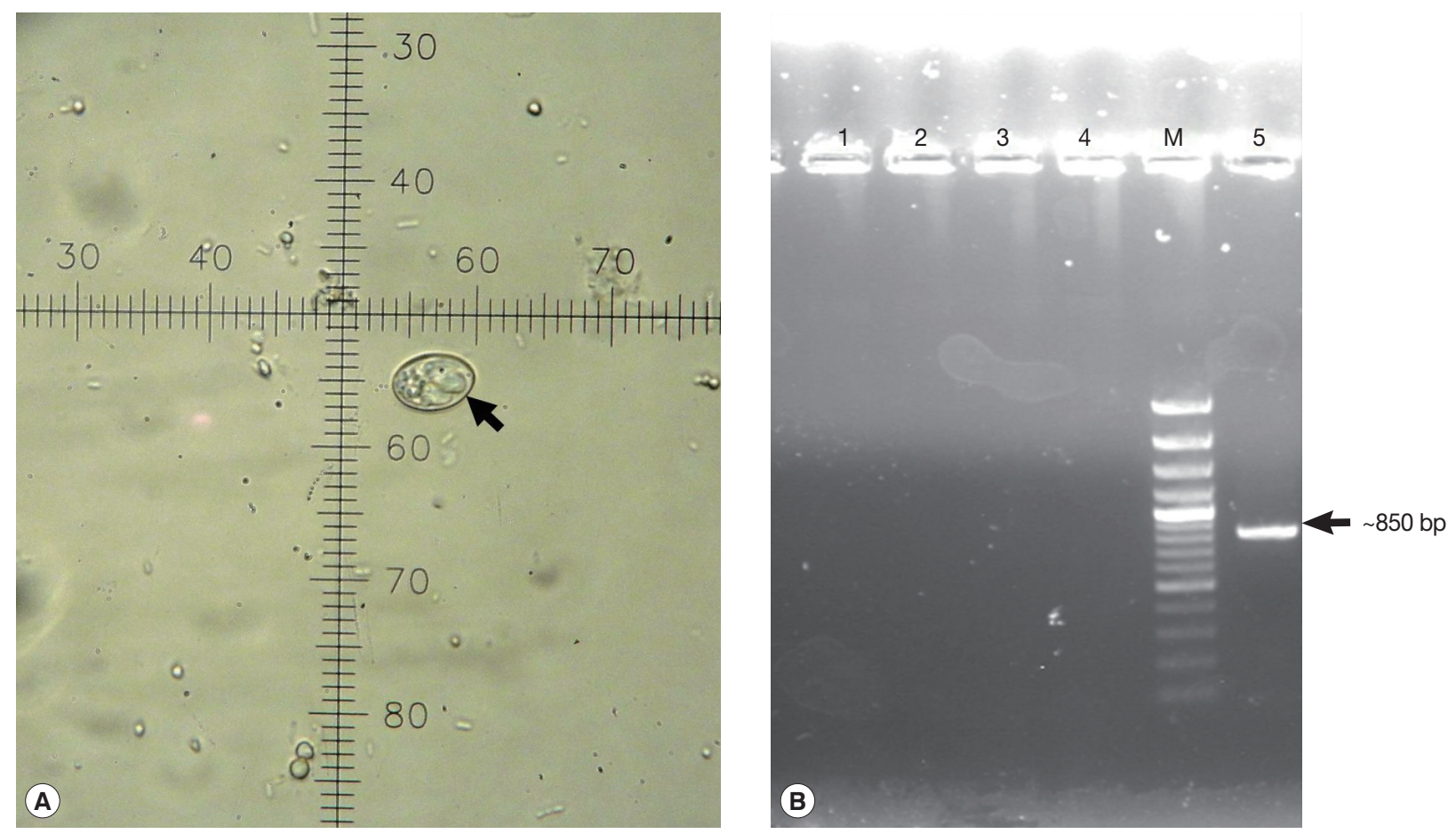

Fig. 2. Identification of Sarcocystis cruzi sporocyst (arrow). (A) Sarcocystis cruzi sporocyst in an AIDS patient's diarrheal sample. 1 ocular unit=2.5 $\mu \mathrm{m}$. (B) Agarose gel of semi-nested PCR products. Arrow indicates Sarcocystis cruzi DNA ( 850 bp). Lane M, 100 bp molecular size marker; lanes 1-2, microscopically-negative stool specimens; lane 3, Cystoisospora belli sample; lane 4, negative control; lane 5 , this case. 
dilation of the bile ducts or gallbladder. During the last episode of diarrhea, the patient was referred to the author's laboratory where numerous oocysts of Cystoisospora belli and sporocysts of Sarcocystis were identified by parasitological stool examinations. The patient was treated with cotrimoxazole and showed improvements in 4 days.

Examples of immunodeficient individuals include those with AIDS, cancer, and transplant patients who are taking certain immunosuppressive drugs; and those with inherited diseases that affect the immune system (e.g., congenital IgA deficiency, congenital agammaglobulinemia). Although all of the enteric conventional pathogens can cause diarrhea in the immunodeficient host, these patients are at risk of developing infections with opportunistic parasites [10,11]. The diagnostic workup of diarrhea in an immunocompromised or immunosuppressed host can be more difficult. For parasitic and coccidial infections, including Cryptosporidium, C. belli, C. cayetanensis, and microsporidia, special stains, including modified acid-fast, are needed.

Unfortunately, the diarrhea caused by these parasites cannot be differentiated from that caused by other enteric pathogens, such as enterotoxigenic Escherichia coli, Giardia lamblia, and viral agents (Norwalk and rotavirus) [10]. Identification of these parasites will help the proper therapy of these patients, because effective drugs are available for the treatment of most of these opportunistic infections [10].

Studies from some other regions of Iran, similar to what was found in our study (Table 1) demonstrated that cryptosporidiosis is a common opportunistic enteric protozoan disease encountered in HIV/AIDS-infected individuals with $\mathrm{CD} 4^{+}$counts $<200$ cells [12]. Up to now, few researchers have reported $C$. belli infection in immunosuppressed patients in Iran $[13,14]$. However; there is no report available on the prevalence of $C$. belli infection in Iran. The low prevalence of cystoisosporiasis and cyclosporiasis in Iran and especially in this research may be related to the secondary prophylaxis for pneumocystosis through the administration of cotrimoxazole during the course of AIDS and use of immunosuppressive agents for solid organ or bone marrow transplantation, since $C$. belli and $C$. cayetanensis are sensitive to this treatment. Very limited information is available on microsporidial infection from Iran. The prevalence of intestinal microsporidial infection depends on geographical area, diagnostic methods, and immune status of study population [15].

Sarcocystis species have global distribution. To date, humans are an intermediate host of some unknown Sarcocystis spp. and a definitive host of 2 known species, Sarcocystis hominis (syn. S. bovihominis) with a cattle-man cycle and Sarcocystis suihominis with a pig-man cycle $[6,7]$. The standard method for diagnosing intestinal sarcocystosis is usually made by microscopically identifying typical Sarcocystis sporocysts each with 4 sporozoites in stool samples. Because parasites may be difficult to find in light infections, patients might need to submit several specimens collected on different days and a concentration procedure is recommended [6]. The prevalence of intestinal sarcocystosis in humans has been reported to be between $1.1 \%$ to $10.4 \%$ in some European countries, $0.5 \%$ in Australia, $0 \%$ in Argentina, and between $0.4 \%$ and $23.2 \%$ in Tibet and Thailand, respectively, although reports of human enteric infections with Sarcocystis are limited [10]. High prevalence rate of intestinal Sarcocystis infection in northern Thailand was likely due to risk factors, including the local habitat of eating raw or undercooked beef and pork, poor living conditions, and low levels of hygiene in Thai laborers [16]. Testing for intestinal sarcocystosis is not routinely done in most Iranian clinical laboratories, even when stool is tested for ova and parasites, so there are no data on the presence of intestinal sarcocystosis in Iranian individuals. In spite of high percentages of muscular sarcocystosis in slaughtered livestock in Iran, especially Fars province [17-23], the very low prevalence rate of Sarcocystis in this study is indicative of the religious habit of eating properly cooked beef in immunodeficient patients. Because of the Islamic prohibition on eating porcine, there is no pig farming in Iran, so the transmission of Sarcocystis through pork is very unlikely. Unlike the morphology of sarcocysts, differentiation of Sarcocystis species from one another is not possible only by morphology or size of sporocysts or oocysts, although the oval sporocysts belonging to cats measure about $12 \mu \mathrm{m}$ and in dogs and humans about $15 \mu \mathrm{m}$ [24].

The $18 \mathrm{~S}$ ribosomal DNA region has been extensively used as a suitable target to differentiate Sarcocystis spp. and phylogenetic relationship among them [25-28]. The method used in this study for identification was achieved by $18 \mathrm{~S}$ rDNA amplification, sequencing, and comparing with the key reference sequences of Sarcocystis species deposited in the GenBank. Comparing the molecular sequencing results of the sample obtained in the present study with those previously reported for different Sarcocystis spp. revealed the positive Sarcocystis sample as $S$. cruzi. The nucleotide sequence of $S$. cruzi obtained in this study was deposited in the GenBank database under accession 


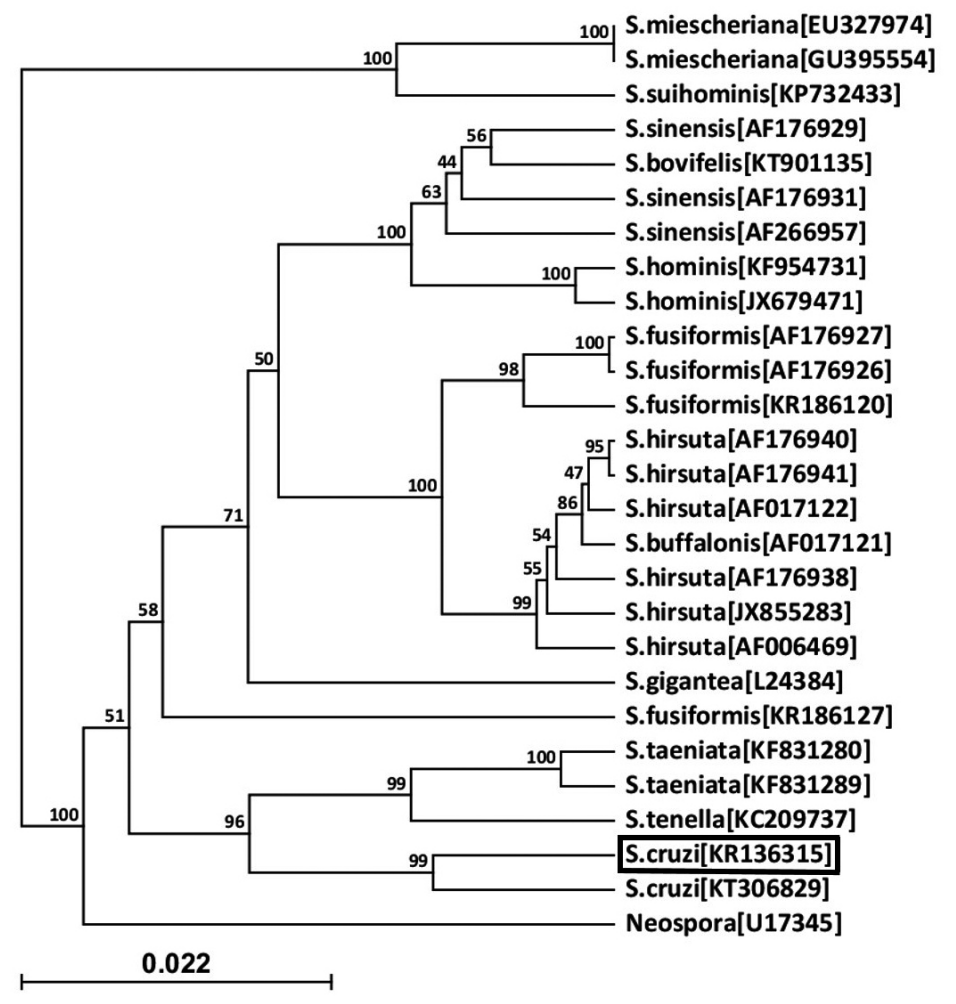

Fig. 3. A phylogenic tree of Sarcocystis cruzi (KR136315) with other Sarcocystis species. S. cruzi of this case is boxed. Neospora caninum was used as an outgroup. The relationships were inferred by phylogenetic analysis of partial 18S rDNA sequence data using CLC Sequence Viewer 6 software (http://www.clcbio.com). Accession no. of sequence is given in bracket. The scale bar indicates distance.

no. KR136315. This sequence has 100\%, 99\%, and 97\% homology compared to $S$. cruzi with accession nos. KT306829, AF176933, and KT306829, which are species using cattle and water buffaloes as intermediate hosts, respectively. The phylogenetic analysis based on CLC Sequence Viewer 6 software (Fig. 3) indicated 5 distinct clades in the genus Sarcocystis. S. cruzi from the present study which was placed in a group with S. cruzi (accession no. KT306829) from Punjab, India. This group was adjacent to other groups containing $1 S$. tenella and $2 S$. taeniata sequences. This issue indicates that $S$. tenella and $S$. taeniata have the closest relationship with S. cruzi compared with other species in the genus Sarcocystis.

To the best of our knowledge, this is the first report of intestinal sarcocystosis caused by $S$. cruzi from a naturally infected human, an AIDS patient in Iran. It can be concluded that Sarcocystis species present in an enteric from of infection in humans are not restricted to the 2 previously established species, namely S. hominis and S. suihominis.

\section{ACKNOWLEDGMENT}

We are grateful to Dr. Seyed Mohammad Hossein Afsarian (from Microbiology Department, Fasa University of Medical Sciences) for his assistance in drawing phylogenetic tree.

\section{CONFLICT OF INTEREST}

There is no conflict of interest related to this study.

\section{REFERENCES}

1. Dwivedi KK, Prasad G, Saini S, Mahajan S, Lal S, Baveja UK. Enteric opportunistic parasites among HIV infected individuals: associated risk factors and immune status. Jpn J Infect Dis 2007; 60: 76-81.

2. Agholi M, Hatam GR, Motazedian MH. HIV/AIDS-associated opportunistic protozoal diarrhea. AIDS Res Hum Retroviruses 2013; 29: 35-41.

3. Agholi M, Hatam GR, Motazedian MH. Microsporidia and coccidia as causes of persistence diarrhea among liver transplant 
children: incidence rate and species/genotypes. Pediatr Infect Dis J 2013; 32: 185-187.

4. Bhaijee F, Subramony C, Tang SJ, Pepper DJ. Human immunodeficiency virus-associated gastrointestinal disease: common endoscopic biopsy diagnoses. Pathol Res Int 2011; 2011: 247923.

5. Stark D, Barratt JL, van Hal S, Marriott D, Harkness J, Ellis JT. Clinical significance of enteric protozoa in the immunosuppressed human population. Clin Microbiol Rev 2009; 22: 634650.

6. Fayer R, Esposito DH, Dubey JP. Human infections with Sarcocystis species. Clin Microbiol Rev 2015; 28: 295-311.

7. Poulsen CS, Stensvold CR. Current status of epidemiology and diagnosis of human sarcocystosis. J Clin Microbiol 2014; 52: 3524-3530.

8. Agholi M, Heidarian HR, Moghadami M, Hatam GR. First detection of acalculous cholecystitis associated with Sarcocystis infection in a patient with AIDS. Acta Parasitol 2014; 59: 310-315.

9. Xiang Z, Chen X, Yang L, He Y, Jiang R, Rosenthal BM, Luan P, Attwood SW, Zuo Y, Zhang YP, Yang Z. Non-invasive methods for identifying oocysts of Sarcocystis spp. from definitive hosts. Parasitol Int 2009; 58: 293-296.

10. Marcos LA, Gotuzzo E. Intestinal protozoan infections in the immunocompromised host. Curr Opin Infect Dis 2013; 26: 295-301.

11. Danziger-Isakov L. Gastrointestinal infections after transplantation. Curr Opin Gastroenterol 2014; 30: 40-46.

12. Daryani A, Sharif M, Meigouni M, Mahmoudi FB, Rafiei A, Gholami Sh, Khalilian A, Gohardehi Sh, Mirabi AM. Prevalence of intestinal parasites and profile of $\mathrm{CD}^{+}$counts in $\mathrm{HIV}^{+}$/AIDS people in north of Iran, 2007-2008. Pak J Biol Sci 2009; 12: 1277-1281.

13. Shafiei R, Najjari M, Kargar Kheirabad A, Hatam G. Severe diarrhea due to Cystoisospora belli infection in an HTLV-1 woman. Iran J Parasitol 2016; 11: 121-125.

14. Nateghi Rostami M, Nikmanesh B, Haghi-Ashtiani MT, Monajemzadeh M, Douraghi M, Ghalavand Z, Kashi L. Isospora belli associated recurrent diarrhea in a child with AIDS. J Parasit Dis 2014; 38: 444-446.

15. Mirjalali H, Mirhendi H, Meamar AR, Mohebali M, Askari Z, Mirsamadi ES, Rezaeian M. Genotyping and molecular analysis of Enterocytozoon bieneusi isolated from immunocompromised patients in Iran. Infect Genet Evol 2015; 36: 244-249.

16. Wilairatana P, Radomyos P, Radomyos B, Phraevanich R, Plooksawasdi W, Chanthavanich P, Viravan C, Looareesuwan S. Intestinal sarcocystosis in Thai laborers. Southeast Asian J Trop Med
Public Health 1996; 27: 43-46.

17. Oryan A, Moghaddar N, Gaur SN. The distribution pattern of Sarcocystis species, their transmission and pathogenesis in sheep in Fars Province of Iran. Vet Res Commun 1996; 20: 243-453.

18. Shekarforoush SS, Razavi SM, Dehghan SA, Sarihi K. Prevalence of Sarcocystis species in slaughtered goats in Shiraz, Iran. Vet Rec 2005; 156: 418-420.

19. Nourollahi-Fard SR, Kheirandish R, Sattari S. Prevalence and histopathological finding of thin-walled and thick-walled Sarcocysts in slaughtered cattle of Karaj abattoir, Iran. J Parasit Dis 2015; 39: 272-275.

20. Nourani H, Matin S, Nouri A, Azizi H. Prevalence of thin-walled Sarcocystis cruzi and thick-walled Sarcocystis hirsuta or Sarcocystis hominis from cattle in Iran. Trop Anim Health Prod 2010; 42: 1225-1227.

21. Nourollahi Fard SR, Asghari M, Nouri F. Survey of Sarcocystis infection in slaughtered cattle in Kerman, Iran. Trop Anim Health Prod 2009; 41: 1633-1636.

22. Shekarforoush SS, Razavi SM, Dehghan SA, Sarihi K. Prevalence of Sarcocystis species in slaughtered goats in Shiraz, Iran. Vet Rec 2005; 156: 418-420.

23. Oryan A, Ahmadi N, Mousavi SM. Prevalence, biology, and distribution pattern of Sarcocystis infection in water buffalo (Bubalus bubalis) in Iran. Trop Anim Health Prod 2010; 42: 1513-1518.

24. Rommel M. Sarcocystosis of domestic animals and humans. In Pract 1985; 7: 158-160.

25. Gjerde B, Hilali M, Mawgood SA. Molecular characterisation of three regions of the nuclear ribosomal DNA unit and the mitochondrial cox1 gene of Sarcocystis fusiformis from water buffaloes (Bubalus bubalis) in Egypt. Parasitol Res 2015; 114: 3401-3413.

26. Coelho C, Gomes J, In Jcio J, Amaro A, Mesquita JR, Pires I, Lopes AP, Vieira-Pinto M. Unraveling Sarcocystis miescheriana and Sarcocystis suihominis infections in wild boar. Vet Parasitol 2015; 212: $100-104$.

27. Dubey JP, Sykes JE, Shelton GD, Sharp N, Verma SK, Calero-Bernal R, Viviano J, Sundar N, Khan A, Grigg ME. Sarcocystis caninum and Sarcocystis svanai n. spp. (Apicomplexa: Sarcocystidae) associated with severe myositis and hepatitis in the domestic dog (Canis familiaris). J Eukaryot Microbiol 2015; 62: 307-317.

28. Calero-Bernal R, Verma SK, Oliveira S, Yang Y, Rosenthal BM, Dubey JP. In the United States, negligible rates of zoonotic sarcocystosis occur in feral swine that, by contrast, frequently harbour infections with Sarcocystis miescheriana, a related parasite contracted from canids. Parasitology 2015; 142: 549-556. 